\title{
Superior Gluteal Reconstruction Results in Promising Outcomes for Massive Abductor Tendon Tears
}

\author{
Robert B. Browning, M.D., Ian M. Clapp, M.S., Thomas D. Alter, M.S., \\ Benedict U. Nwachukwu, M.D., M.B.A., Theodore Wolfson, M.D., M.B., \\ Sunikom Suppauksorn, M.D., and Shane J. Nho, M.D., M.S.
}

\begin{abstract}
Purpose: To evaluate the l-year outcomes of a small patient series following open gluteus medius/minimus repair with human dermal allograft incorporated into the repair construct using a double-row repair. Methods: Data from consecutive patients undergoing a superior gluteal reconstruction for massive, irreparable abductor tendon tears with severe tendon loss and atrophy by a single fellowship trained surgeon from January 2018 to May 2019 were collected and analyzed. Baseline demographic data and magnetic resonance imaging were collected preoperatively. Clinical outcomes including Hip Outcome Score-Activities of Daily Living (HOS-ADL), HOS-Sports Subscale (HOS-SS), modified Harris hip score (mHHS), international Hip Outcome Score-12 (iHOT-12), visual analog scale (VAS) pain, and VAS satisfaction were recorded at 1-year postoperatively. Results: A total of 8 patients underwent open superior gluteal reconstruction for severe hip abductor deficiency. The mean age and body mass index were $62.6 \pm 7.3$ years and $29.6 \pm 5.3 \mathrm{~kg} / \mathrm{m}^{2}$, respectively. The majority of patients were female $(\mathrm{N}=7,87.5 \%)$. Three $(37.5 \%)$ patients had undergone previous endoscopic gluteus medius repair and presented for revision surgery. All patients had full-thickness tears with gluteus medius and gluteus minimus involvement. Patients were evaluated at an average of $11.5 \pm 1.7 \mathrm{months}$ from the initial surgical intervention and reported a mean HOS-ADL of 82.9 \pm 24.3 , HOS-SS of 73.2 \pm 37.3 , mHHS of 83.6 \pm 17.1 , iHOT12 of $63.9 \pm 27.4$, VAS Pain of $30.0 \pm 23.1$, and VAS Satisfaction of $87.1 \pm 17.0$. There was no evidence of retears in this patient cohort as defined by physical examination findings and/or corroborating magnetic resonance imaging. Conclusions: Superior gluteal reconstruction for massive, irreparable abductor tendon tears with severe tendon loss and atrophy is a technique that demonstrates promising l-year postoperative outcomes in both primary and revision patients. Level of Evidence: Level IV, therapeutic case series.
\end{abstract}

A bductor tendinopathy of the hip has become an increasingly recognized cause of lateral hip pain and dysfunction and a major contributor to greater trochanteric pain syndrome. Many patients who have been previously diagnosed with trochanteric bursitis are now recognized to have abductor tendinopathy, which can be refractory to nonsurgical treatment. ${ }^{1}$ Gluteus medius and minimus tendinopathy of the hip encompasses a broad spectrum of degenerative tendinopathy

From the Department of Orthopaedic Surgery, Division of Sports Medicine, Rush University Medical Center, Chicago, Illinois (R.B.B., I.M.C., T.D.A., T.W., S.S., S.J.N.); and Department of Orthopaedic Surgery, Hospital for Special Surgery, New York, New York (B.U.N.), U.S.A.

The authors report the following potential conflicts of interest or sources of funding: B.U.N. reports financial support from Remote Health, outside the submitted work. S.J.N. receives nonfinancial support from AlloSource; other from the American Orthopaedic Association and American Orthopaedic Society for Sports Medicine; nonfinancial support from Arthrex; other from the Arthroscopy Association of North America; nonfinancial support from Athletico, DJ Orthopaedics, Linvatec, and Miomed; personal fees from Ossur; nonfinancial support from Smith $\theta$ Nephew; and personal fees from Springer ranging from interstitial, partial-thickness tears to retracted, full-thickness tears. ${ }^{2}$ Abductor tendon tears have been referred to as "rotator cuff tears of the hip," due to similarities of the gluteus medius and the gluteus minimus tears to supraspinatus and infraspinatus tears, respectively. ${ }^{3,4}$ Magnetic resonance imaging (MRI) has shown to be an accurate imaging modality with excellent interobserver reliability for the diagnosis of gluteus medius and minimus tendinopathy and is now

and Stryker, outside the submitted work. Full ICMJE author disclosure forms are available for this article online, as supplementary material.

Received August 7, 2020; accepted May 19, 2021

Address correspondence to Ian Clapp, M.S., Department of Orthopedic Surgery, Rush University Medical Center, 1611 W Harrison St., Suite 300, Chicago,IL 60612.E-mail: Nho.research@rushortho.com

(C) 2021 THE AUTHORS. Published by Elsevier Inc. on behalf of the Arthroscopy Association of North America. This is an open access article under the CC BY-NC-ND license (http://creativecommons.org/licenses/by-nc-nd/4.0/). 2666-061X/201368

https://doi.org/10.1016/j.asmr.2021.05.013 
commonly used in clinical practice for the workup of lateral hip pain and dysfunction. ${ }^{5-9}$ Endoscopic repair of gluteus medius tears has been well-established within the literature, and outcomes have been excellent with medium- to long-term follow-up. ${ }^{10-13}$

Despite promising results with primary repair of torn gluteus tendons, patients with increased fatty infiltration or muscular delamination, specifically in grade 3 and 4 tears (modified Goutallier-Fuchs classification), have reported worse functional outcomes following endoscopic repair. ${ }^{14,15}$ For this reason, alternative treatments have been proposed, including muscle transfers, Achilles allograft procedures, and primary repairs with allograft augmentation. Muscle transfers have reported good-to-excellent early outcomes ${ }^{16-20}$; however, this nonanatomic procedure may have increased morbidity leading to reduced functional benefit, relegating it to a salvage procedure. ${ }^{21,22} \mathrm{~A}$ few case series reporting the use of synthetic, allograft, and autograft tissue for augmentation or reconstruction have provided positive early results. ${ }^{23-28}$ Suppauksorn et al. ${ }^{29}$ proposed a superior gluteal reconstruction (SGR), which uses an acellular dermal allograft matrix for reconstruction of the massive, irreparable hip abductor tendon tear and is the senior author's technique of choice for large, irreparable gluteus medius tears.

The purpose of this study was to evaluate the 1-year outcomes of a small patient series following open gluteus medius/minimus repair with human dermal allograft incorporated into the repair construct using a double-row repair. We hypothesized that patients undergoing SGR would have favorable patient-reported outcomes (PROs) without evidence of clinical retears at 1-year follow-up.

\section{Methods}

\section{Preoperative Evaluation and Clinical Indications}

After we obtained institutional review board approval, clinical data were retrospectively retrieved from a prospectively maintained institutional surgical repository. Patients who underwent gluteus medius and/or minimum tendon repair with the technique of SGR done by a single fellowship-trained surgeon (S.J.N.) between December 2018 and August 2019 were identified. All patients were assessed by the senior author (S.J.N.) diagnosed with abductor tendon tear had symptoms of lateral hip pain, tenderness on palpation of the greater trochanter, weakness with resisted hip abduction, and findings on MRI of gluteus medius and/or minimus tear. Patients indicated for operative repair had not responded to a trial of conservative management for a minimum of 3 months with a combination of activity modification, oral antiinflammatory medications, and a focused physical therapy regimen. The exclusion criterion was patients undergoing endoscopic gluteus medius repair. In addition, no patients had a total hip arthroplasty in place.

\section{Radiographic Measurements}

All patients underwent preoperative MRIs. Imaging was analyzed by a fellowship-trained orthopaedic surgeon for gluteus medius and minimus involvement, tear type, retraction, presence of trochanteric bursitis, the Goutallier-Fuchs classification grade, ${ }^{30}$ and tensor fascia latae hypertrophy.

\section{Operative Technique}

The SGR technique has been previously described in the literature. The indications for SGR include failure of primary gluteal repair, massive tears with fatty atrophy, or muscular delamination from gluteal tendons (Fig 1). ${ }^{29}$ The procedure was performed using a direct lateral approach with the patient in the lateral decubitus position. The iliotibial band was incised in line with their fibers to allow entry into the peritrochanteric space, and a trochanteric bursectomy was performed. The greater trochanter was visualized and insertional anatomy of the abductor tendons defined. The torn edges of the gluteus medius and minimus tendons were identified and debrided while as much viable tendon as

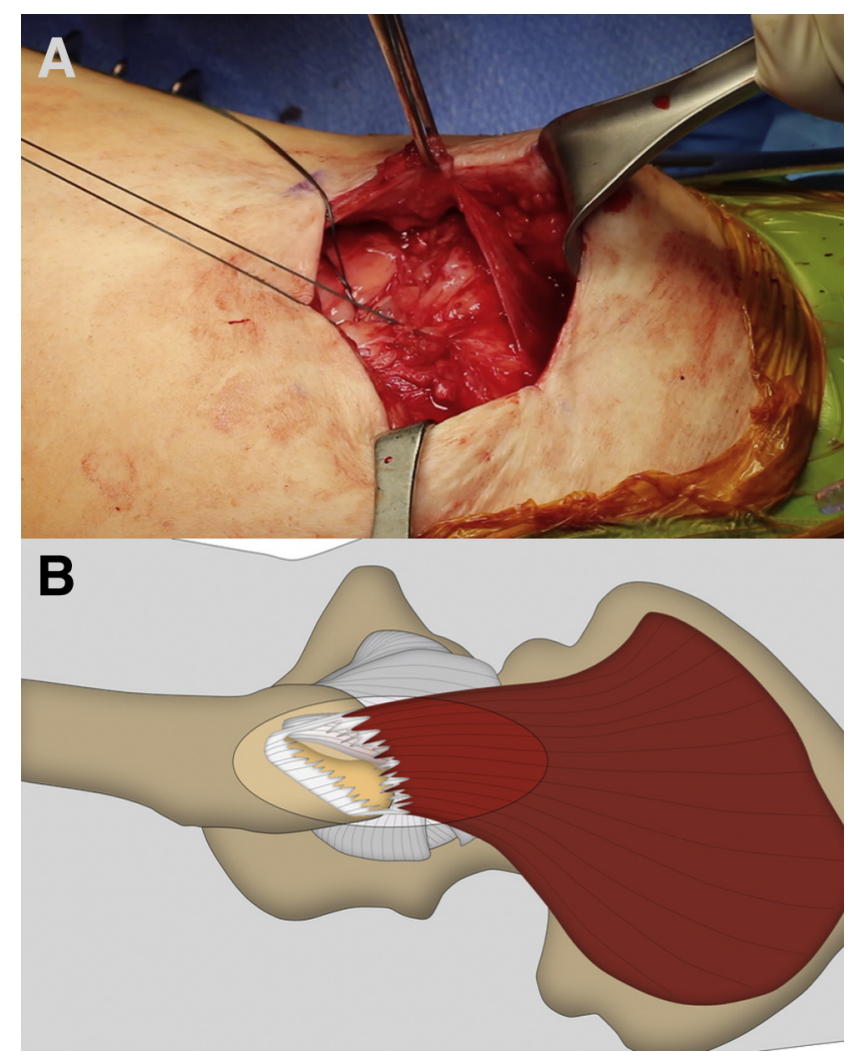

Fig 1. An image (A) and an illustration (B) of a massive gluteus medius/minimus tear of left hip with sutures placed on either side of the tear aiding in visualization before repair. 

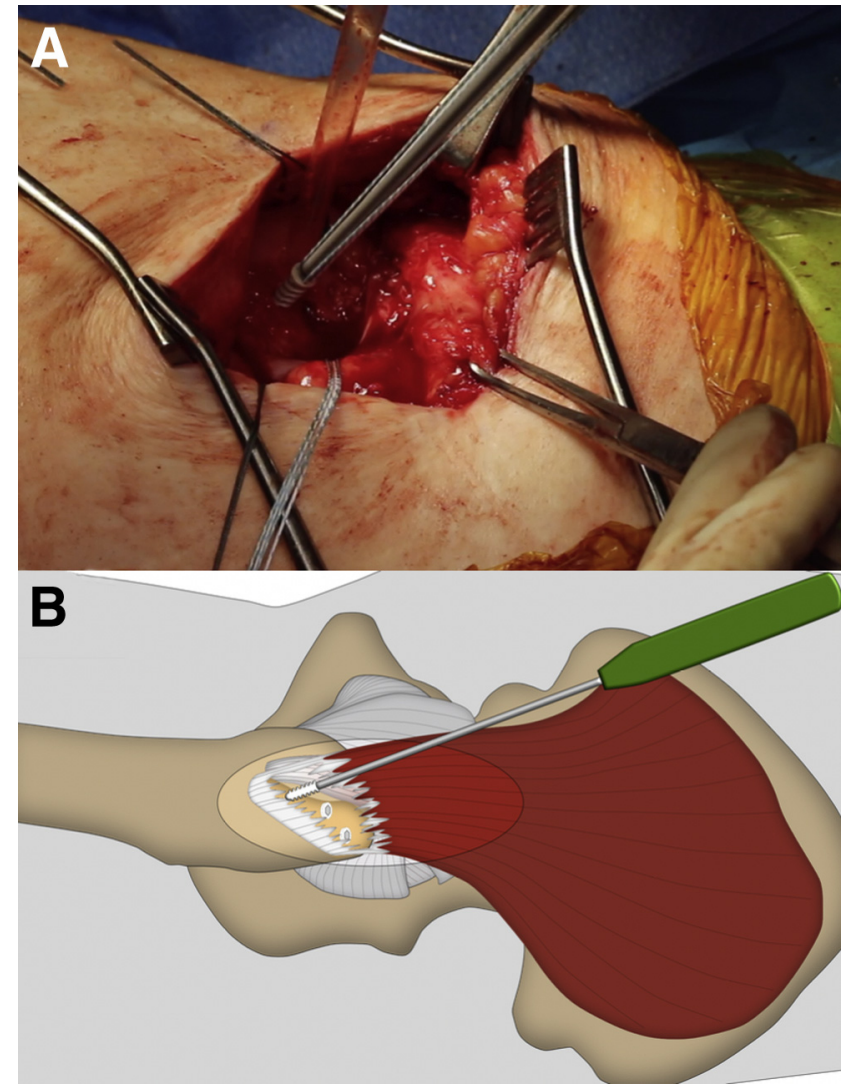

Fig 2. An image (A) and an illustration (B) of the placement of PEEK anchors in a left hip.

possible was preserved. The gluteus minimus was mobilized by freeing the tendon from the underlying capsule and overlying tensor fascia latae. The insertion site on the greater trochanter was then prepared. Anchor configuration for a double-row transosseous equivalent repair was planned based on the tear morphology to recreate the native tendon insertion. Two medial-row 4.75-mm fully threaded PEEK (polyether ether ketone) anchors (SwiveLock; Arthrex, Naples, FL) were first inserted in the lateral facet and superoposterior facet near the medial border of the footprint (Fig 2). The number of suture anchors depends on the size of the tear. The sutures and tapes were then passed through the proximal margin of the tendon in a horizontal mattress configuration with use of a free needle. The acellular human dermal allograft matrix patch (AlloMend; AlloSource, Centennial, CO) was trimmed to the appropriate dimensions and placed over the tendon defect and footprint. With the hip in $20^{\circ}$ of abduction and neutral rotation, the medial-row sutures and tapes were then passed vertically through the graft and tied to compress the patch to the native tendons (Fig 3). One limb from each pair of sutures (4 pairs) was then incorporated into 2 lateral-row 4.75mm PEEK anchors (SwiveLock; Arthrex). The sutures were sequentially tensioned before anchor insertion to compress the graft-tendon unit to the footprint and complete the double-row SGR (Fig 4). The integrity of the final reconstruction was assessed with gentle passive rotation of the hip, and following irrigation, the iliotibial band and superficial wound were closed in standard layered fashion.

\section{Postoperative Rehabilitation}

Following SGR, patients began a 4-phase rehabilitation protocol. For the first 8 weeks following surgery, patients were restricted to $20 \mathrm{lbs}$ of foot flat weightbearing to minimize joint reactive forces. During this period, patients ambulated with an assistive device. A continuous passive motion device was set at $30^{\circ}$ to $70^{\circ}$ of flexion, and was increased by $5^{\circ}$ increments until $0^{\circ}$ to $90^{\circ}$ was achieved at 2 weeks following surgery. Patients were fitted with a postoperative hip brace and no active hip abduction or internal rotation and no passive hip adduction or external rotation was permitted for 6 to 8 weeks. Phase 2 began at 6 weeks and progressed the patient to full weight-bearing and initiated hip-strengthening exercises as the brace was discontinued. Phase 3 began at 12 weeks and allowed for ambulation without assist and return as tolerated to general activity. At 24 weeks patients entered phase 4,
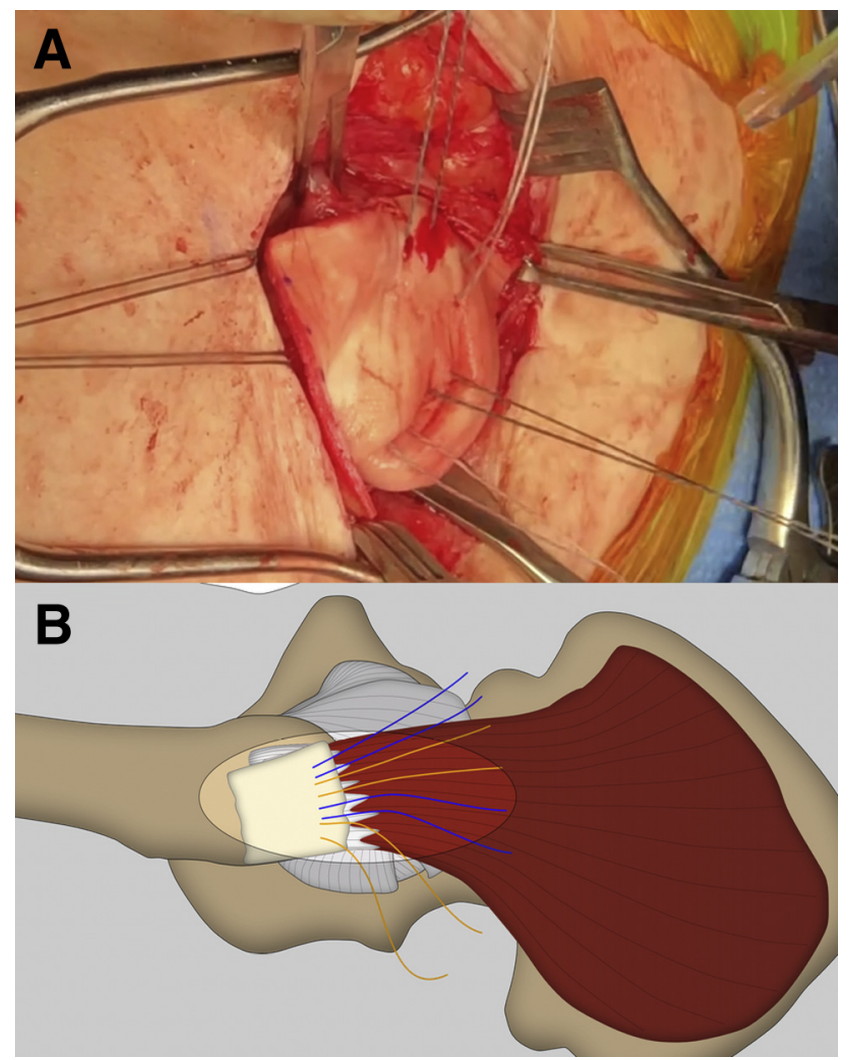

Fig 3. An image (A) and illustration (B) of the acellular human dermal allograft matrix patch (AlloMend; AlloSource) placed over the tendon defect and the greater trochanter of a left hip. The sutures are passed using free needle through the proximal tendon and the overlying graft in horizontal mattress fashion. 


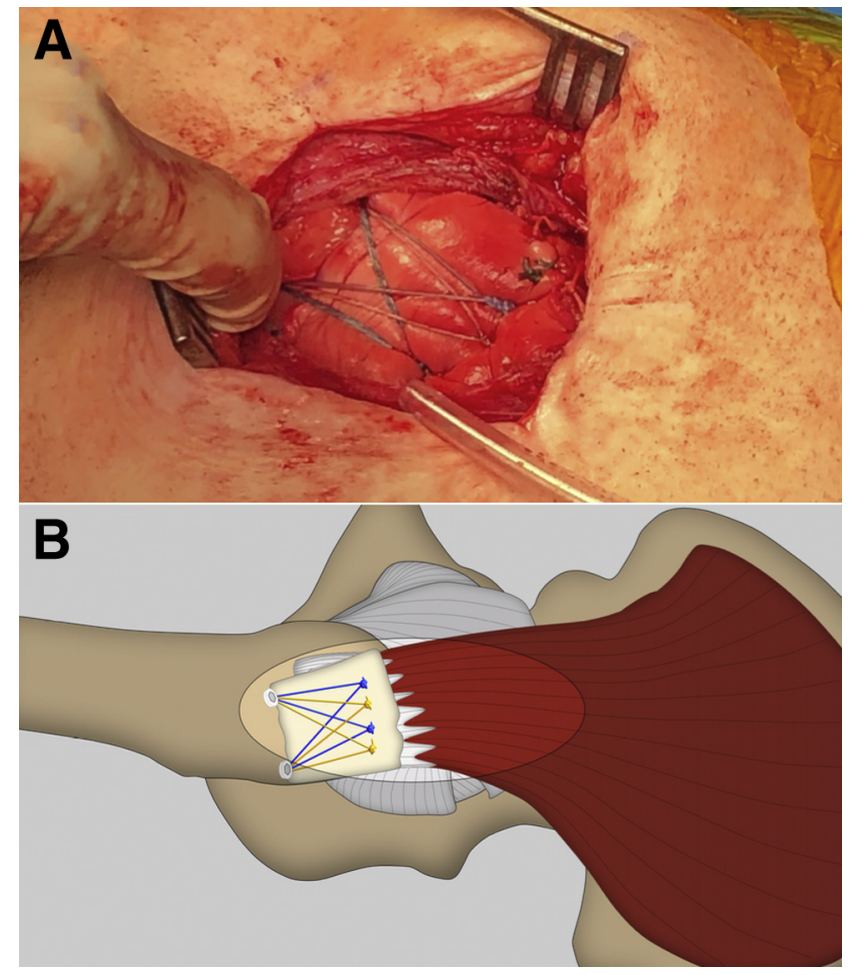

Fig 4. An image (A) and an illustration (B) of the complete double-row suture repair with superior gluteal reconstruction of a left hip using acellular human dermal allograft matrix patch (AlloMend; AlloSource).

which focused on strength, endurance, plyometric progression, initiation of a running program, and sportspecific drills. Patients were cleared to discontinue physical therapy and return to activity or sport at 4 to 6 months depending on progress.

\section{Functional Outcome Measures}

Preoperatively, demographic data were collected from all patients, including sex, age, operative extremity, body mass index, duration of symptoms, and comorbidities. Patients completed l-year postoperative hipspecific PRO instruments including the Hip Outcome Score-Activities of Daily Living Subscale (HOS-ADL), ${ }^{31}$ HOS-Sports Specific Subscale (HOS-SS), the modified Harris Hip Score (mHHS), ${ }^{32}$ and the international Hip Outcome Tool-12.33,34 Patients also received VAS satisfaction level and VAS pain at 2-years postoperatively. To determine whether patients achieved a clinically significant outcome, the patient acceptable symptomatic state (PASS) was used. Okoroha et al. ${ }^{35}$ calculated PASS for patients undergoing endoscopic gluteus medius repair for the HOS-ADL, HOS-SS, and mHHS, and the threshold values were 77.9, 56.9, and 69.3 , respectively.

\section{Statistics Analysis}

Noncontinuous variables are reported as frequency statistics, whereas descriptive statistics for all continuous variables are reported as mean and standard deviations (SDs). The percentage of patients achieving PASS for the HOS-ADL, HOS-SS, and mHHS was calculated.

\section{Results}

\section{Demographics}

A total of 8 patients underwent open SGR for severe hip abductor deficiency during the study period and were evaluated at an average of $11.5 \pm 1.7$ months from initial surgical intervention. The mean age and body mass index were $62.6(\mathrm{SD} \pm 7.3$ ) years and 29.6 $(\mathrm{SD} \pm 5.3)$ respectively, and the majority of patients were female $(\mathrm{N}=7,87.5 \%)$. The characteristics of the patients are shown in Table 1. The patients' physical examination findings are shown in Table 2.The MRI characteristics are reported in Table 3. Eight $(100 \%)$ patients had full-thickness tears with gluteus medius and minimus involvement with retraction present.

\section{Intraoperative Data}

All patients underwent open gluteus medius/minimus repair with allograft augmentation. One patient underwent a concomitant gluteus maximus transfer at time of surgery. Three patients $(37.5 \%)$ had undergone previous primary endoscopic gluteus medius/minimus repair without augmentation and presented with evidence of retears and underwent revision surgery.

\section{Clinical Outcomes}

Postoperative PROs for the study population are reported in Table 4 . Briefly, at 1-year follow-up, patients had an average HOS-ADL of $82.9 \pm 24.3$, HOS-SS of 73.2 \pm 37.3 , mHHS of $83.6 \pm 17.1$, international Hip Outcome Tool- 12 of $63.9 \pm 27.4$, VAS Pain of $30.0 \pm 23.1$, and VAS Satisfaction of $87.1 \pm 17.0$. The percentage of patients achieving PASS for the HOS-ADL, HOS-SS, and mHHS were $62.5 \%, 50.0 \%$, and $75.0 \%$, respectively.

\section{Postoperative Period}

One patient underwent open repair of the contralateral gluteus medius during the follow-up period.

Table 1. Patient Demographics $(n=8)$

\begin{tabular}{lc}
\hline Sex $(\%$ Female $)$ & $7(87.5 \%)$ \\
Age, $\mathrm{y}$ & $62.6 \pm 7.3(55-77)$ \\
BMI $\left(\mathrm{kg} / \mathrm{m}^{2}\right)$ & $29.6 \pm 5.3(20.8-33.7)$ \\
Laterality (right hip) & $5(62.5 \%)$ \\
Smoking status & $0(0.0 \%)$ \\
Duration of symptoms before surgery & \\
$1(<4 \mathrm{mo})$ & $0(0.0 \%)$ \\
$2(4-12 \mathrm{mo})$ & $1(12.5 \%)$ \\
$3(1-2 \mathrm{y})$ & $3(37.5 \%)$ \\
$4(>2 \mathrm{y})$ & $4(50.0 \%)$ \\
\hline NOTE. Results reported as $\mathrm{N}(\%)$ or mean \pm standard deviation \\
(range). \\
BMI, body mass index.
\end{tabular}


Table 2. Preoperative Physical Examination

\begin{tabular}{ll}
\hline Flexion & $106.3 \pm 10.6(90-120)$ \\
IR at $90^{\circ}$ & $27.5 \pm 7.1(20-40)$ \\
ER at $90^{\circ}$ & $15.0 \pm 8.7(0-25)$ \\
FABER (positive) & $5(62.5 \%)$ \\
FADIR (positive) & $3(37.5 \%)$ \\
Painful arc from 1-3 o'clock (positive) & $8(100 \%)$ \\
Log roll (positive) & $0(0.0 \%)$ \\
Circumduction clunk (positive) & $0(0.0 \%)$ \\
Trochanteric pain (positive) & $8(100 \%)$ \\
Pain with abduction (positive) & $8(100 \%)$ \\
Abduction strength & $4.0 \pm 0.71(4-5)$ \\
Limp (present) & $4(50 \%)$
\end{tabular}

NOTE. Results reported as $\mathrm{N}(\%)$ or mean \pm standard deviation (range).

ER, external rotation; FABER, flexion abduction, external rotation. FADIR, flexion adduction internal rotation; IR, internal rotation.

Another patient continued to have a limp in the postoperative period. However, this patient developed back pain following postoperative physical therapy and was found to have L4-L5 herniated disk on MRI and underwent an ablation. There was no evidence of clinical retears in any patients as defined by physical examination findings (persistent or recently developed weakness of abductor strength against resistance and/or Trendelenburg sign) and/or corroborating MRI (gold standard for assessing retears), which was indicated with continued or newly acute pain and dysfunction in the postoperative time period.

\section{Discussion}

The most important findings of this study are that at 1 -year follow-up SGR resulted in favorable outcome with $62.5 \%, 50.0 \%$, and $75.0 \%$ of patients achieving

Table 3. Preoperative MRI Characteristics

\begin{tabular}{lc}
\hline Gluteus Medius Involvement & $8(100 \%)$ \\
Gluteus minimus involvement & $8(100 \%)$ \\
Full-thickness tear & $8(100 \%)$ \\
Retraction (present) & $8(100 \%)$ \\
Greater trochanteric bursitis & $1(12.5 \%)$ \\
$\quad$ Mild & $2(25.0 \%)$ \\
Moderate & $5(62.5 \%)$ \\
Severe & \\
Goutallier-Fuchs classification gluteus medius & $1(12.5 \%)$ \\
0 & $2(25.0 \%)$ \\
1 & $3(37.5 \%)$ \\
2 & $1(12.5 \%)$ \\
3 & $1(12.5 \%)$ \\
4 & \\
Goutallier-Fuchs classification gluteus minimus & $1(12.5 \%)$ \\
0 & $0(0.0 \%)$ \\
1 & $4(50.0 \%)$ \\
2 & $1(12.5 \%)$ \\
3 & $2(25.0 \%)$ \\
4 & $4(50.0 \%)$ \\
Tensor fascia latae hypertrophy &
\end{tabular}

MRI, magnetic resonance imaging.
Table 4. 1-Year Postoperative Patient-Reported Outcomes

\begin{tabular}{lr}
\hline HOS-ADL & $82.9 \pm 24.3$ \\
HOS-SS & $73.2 \pm 37.3$ \\
mHHS & $83.6 \pm 17.1$ \\
iHOT-12 & $63.9 \pm 27.4$ \\
VAS Pain & $30.0 \pm 23.1$ \\
VAS Satisfaction & $87.1 \pm 17.0$ \\
\hline \multicolumn{1}{l}{ HOS-ADL, Hip Outcome Score-Activities of Daily Living; HOS-SS, } \\
Hip Outcome Score-Sports Subscale; iHOT-12, International Hip \\
Outcome Tool; mHHS, modified Harris Hip score; VAS, visual analog \\
scale.
\end{tabular}

PASS for the HOS-ADL, HOS-SS, and mHHS, respectively. Furthermore, there was no evidence of clinical retears at final follow-up. Currently, evidence for surgical intervention of abductor tendon tears using synthetic, allograft, and autograft tissue for augmentation of primary gluteus medius repairs is limited.

In the present study, all 8 patients underwent SGR with dermal allograft for massive gluteus medius and minimus tears by the senior author. Patient outcomes at 1 year postoperatively were comparable with outcomes of patients with partial or full-thickness tears who had undergone endoscopic repair, ${ }^{10,13,15}$ with no reported failures. Three $(37.5 \%)$ of the patents in this study underwent SGR for failed endoscopic hip abductor repair. In addition, $7(87.5 \%)$ of these patients had symptoms for greater than 1 year and all had massive gluteus medius and minimus tears. The tensor fascia latae was found to be hypertrophied in $4(50 \%)$ of these patients. This is consistent with an article by Sutter et al., ${ }^{36}$ which explained that the tensor fascia latae is often hypertrophied in the setting of long-term gluteus medius and minimus insufficiency as it adopts a compensatory function in hip abduction.

The chronicity of these tears can lead to a wide separation from the femoral footprint or severe tendon loss. Retraction and the poor quality of the tendon may predispose these patients to retears following surgery, as the repair site may be under high tension. The authors believe that SGR with a acellular augment may strengthen the repair and increase the healing rate. ${ }^{37}$

Davies et al. ${ }^{25}$ described the use of allograft acellular human dermal matrix to augment primary hip abductor tendon repair. In a series of 22 patients undergoing open hip abductor tendon repair, dermal allograft (Graft Jacket; Wright Medical, Memphis, TN) supplemented primary transosseous repair in the 9 patients with grade IV abductor tears (Milwaukee Classification). At 1-year follow-up, 3 (33.3\%) of these patients obtained a poor outcome, with 2 requiring the use of ambulatory aids, compared with no patients with grade I-III tears. ${ }^{25}$ According to the Harris Hip Score, 4 $(50 \%)$ of the patients achieved an "excellent" outcome, which is slightly less than the $50 \%$ to $75 \%$ of patients achieving PASS in the current study. Rao et al. ${ }^{26}$ 
similarly used allograft dermal matrix to augment hip abductor tendon repairs in 12 patients with more promising results. The authors demonstrated significant improvement in pain, abductor strength, gait function, and outcome scores at a mean of 22 months without failure. $^{26}$ The mean Harris Hip Score was 81.26 at follow-up, which is comparable with the mean mHHS at final follow-up in the current cohort. Other authors have described gluteal medius repair with bioinductive patch augmentation using both open and endoscopic techniques. ${ }^{27,28}$

Fehm et al. ${ }^{23}$ described the use of an Achilles tendon allograft with a calcaneal bone block in 7 patients with abductor deficiency following total hip arthroplasty. Patients had an average mHHS of 85.9 at 2-year followup, which is comparable with the average mHHS, 83.6, of the current study. Moreover, the patients demonstrated significant improvement in pain, gait, strength, and subjective outcomes at a minimum of 2-year follow-up. ${ }^{23}$ In patients undergoing abductor tendon suture-anchor repair augmented with a transosseous synthetic scaffold (LARS; Corin Group, Cirencester Cloucestershire, UK), Ebert et al. ${ }^{20,24}$ reported significant improvement in all mean patient PROs and clinical scores, including hip abductor strength and gait performance in 142 patients.

\section{Limitations}

This study has certain limitations that are worth noting. This is only a case series and due to the indication of the surgery, there are a limited number of patients. All patients were based on a single cohort of patients at a large, tertiary care institution who underwent surgery by a single, high-volume surgeon. Therefore, it is possible that these values may not be generalizable to a larger population. In addition, our study findings did not include preoperative PROs, so we are unable to compare pre- and postoperative PROs. However, we applied the principles of the PASS to calculate the percentage of patients achieving a clinically significant outcome. The PASS threshold values were defined in a population of patients undergoing endoscopic gluteus medius repair at 2-year follow-up, and it is possible that the threshold values are not a true representation for patients undergoing SGR at 1-year follow-up.

\section{Conclusions}

SGR for massive, irreparable abductor tendon tears with severe tendon loss and atrophy is a technique that demonstrates promising 1-year postoperative outcomes in both primary and revision patients.

\section{References}

1. Redmond JM, Chen AW, Domb BG. Greater trochanteric pain syndrome. J Am Acad Orthop Surg 2016;24:231-240.
2. Strauss EJ, Nho SJ, Kelly BT. Greater trochanteric pain syndrome. Sports Med Arthrosc Rev 2010;18:113-119.

3. Bunker TD, Esler CN, Leach WJ. Rotator-cuff tear of the hip. J Bone Joint Surg Br 1997;79:618-620.

4. Kagan A 2nd. Rotator cuff tears of the hip. Clin Orthop Relat Res 1999:135-140.

5. Cvitanic O, Henzie G, Skezas N, Lyons J, Minter J. MRI diagnosis of tears of the hip abductor tendons (gluteus medius and gluteus minimus). AJR Am J Roentgenol 2004; 182:137-143.

6. Makridis KG, Lequesne M, Bard H, Djian P. Clinical and MRI results in 67 patients operated for gluteus medius and minimus tendon tears with a median follow-up of 4.6 years. Orthop Traumatol Surg Res 2014;100:849-853.

7. Docking SI, Cook J, Chen S, et al. Identification and differentiation of gluteus medius tendon pathology using ultrasound and magnetic resonance imaging. Musculoskelet Sci Pract 2019;41:1-5.

8. Kingzett-Taylor A, Tirman PF, Feller J, et al. Tendinosis and tears of gluteus medius and minimus muscles as a cause of hip pain: MR imaging findings. AJR Am J Roentgenol 1999;173:1123-1126.

9. Hartigan DE, Perets I, Walsh JP, Domb BG. Imaging of abductor tears: Stepwise technique for accurate diagnosis. Arthrosc Tech 2017;6:e1523-e1527.

10. Hartigan DE, Perets I, Ho SW, Walsh JP, Yuen LC, Domb BG. Endoscopic repair of partial-thickness undersurface tears of the abductor tendon: Clinical outcomes with minimum 2-year follow-up. Arthroscopy 2018;34: 1193-1199.

11. Chandrasekaran S, Gui C, Hutchinson MR, Lodhia P, Suarez-Ahedo C, Domb BG. Outcomes of endoscopic gluteus medius repair: Study of thirty-four patients with minimum two-year follow-up. J Bone Joint Surg Am 2015;97:1340-1347.

12. Perets I, Mansor Y, Yuen LC, Chen AW, Chaharbakhshi EO, Domb BG. Endoscopic gluteus medius repair with concomitant arthroscopy for labral tears: A case series with minimum 5-year outcomes. Arthroscopy 2017;33:2159-2167.

13. Domb BG, Botser I, Giordano BD. Outcomes of endoscopic gluteus medius repair with minimum 2-year follow-up. Am J Sports Med 2013;41:988-997.

14. Thaunat M, Clowez G, Desseaux A, et al. Influence of muscle fatty degeneration on functional outcomes after endoscopic gluteus medius repair. Arthroscopy 2018;34: $1816-1824$.

15. Bogunovic L, Lee SX, Haro MS, et al. Application of the Goutallier/Fuchs rotator cuff classification to the evaluation of hip abductor tendon tears and the clinical correlation with outcome after repair. Arthroscopy 2015;31: 2145-2151.

16. Chandrasekaran S, Darwish N, Vemula SP, Lodhia P, Suarez-Ahedo C, Domb BG. Outcomes of gluteus maximus and tensor fascia lata transfer for primary deficiency of the abductors of the hip. Hip Int 2017;27:567-572.

17. Whiteside LA. Treating abductor deficiency: A transference technique. Orthopedics 201 1;34:e470-e472.

18. Whiteside LA. Surgical technique: Transfer of the anterior portion of the gluteus maximus muscle for abductor 
deficiency of the hip. Clin Orthop Relat Res 2012;470: 503-510.

19. Whiteside LA. Surgical technique: Gluteus maximus and tensor fascia lata transfer for primary deficiency of the abductors of the hip. Clin Orthop Relat Res 2014;472: 645-653.

20. Ebert JR, Smith A, Breidahl W, Fallon M, Janes GC. Association of preoperative gluteal muscle fatty infiltration with patient outcomes in women after hip abductor tendon repair augmented with LARS. Am J Sports Med 2019;47:3148-3157.

21. Chandrasekaran S, Lodhia P, Gui C, Vemula SP, Martin TJ, Domb BG. Outcomes of open versus endoscopic repair of abductor muscle tears of the hip: A systematic review. Arthroscopy 2015;31:2057-67.e2.

22. Grob K, Monahan R, Gilbey H, Ackland T, Kuster MS. Limitations of the vastus lateralis muscle as a substitute for lost abductor muscle function: An anatomical study. J Arthroplasty 2015;30:2338-2342.

23. Fehm MN, Huddleston JI, Burke DW, Geller JA, Malchau H. Repair of a deficient abductor mechanism with Achilles tendon allograft after total hip replacement. J Bone Joint Surg Am 2010;92:2305-2311.

24. Ebert JR, Brogan K, Janes GC. A prospective 2-year clinical evaluation of augmented hip abductor tendon repair. Orthop J Sports Med 2020;8:2325967119897881.

25. Davies JF, Stiehl JB, Davies JA, Geiger PB. Surgical treatment of hip abductor tendon tears. J Bone Joint Surg Am 2013;95:1420-1425.

26. Rao BM, Kamal TT, Vafaye J, Taylor L. Surgical repair of hip abductors. A new technique using Graft Jacket allograft acellular human dermal matrix. Int Orthop 2012;36:2049-2053.

27. Kaplan DJ, Dold AP, Fralinger DJ, Meislin RJ. Endoscopic gluteus medius repair augmented with bioinductive implant. Arthrosc Tech 2016;5:e821-e825.
28. Pascual-Garrido C, Schwabe MT, Chahla J, Haneda M. Surgical treatment of gluteus medius tears augmented with allograft human dermis. Arthrosc Tech 2019;8: e1379-e1387.

29. Suppauksorn S, Nwachukwu BU, Beck EC, Okoroha KR, Nho SJ. Superior gluteal reconstruction for severe hip abductor deficiency. Arthrosc Tech 2019;8:e1255-e1261.

30. Goutallier D, Postel JM, Gleyze P, Leguilloux P, Van Driessche S. Influence of cuff muscle fatty degeneration on anatomic and functional outcomes after simple suture of full-thickness tears. J Shoulder Elbow Surg 2003;12: 550-554.

31. Martin RL, Philippon MJ. Evidence of validity for the hip outcome score in hip arthroscopy. Arthroscopy 2007;23: 822-826.

32. Byrd JW. Hip arthroscopy: Patient assessment and indications. Instr Course Lect 2003;52:711-719.

33. Nwachukwu BU, Chang B, Beck EC, et al. How should we define clinically significant outcome improvement on the iHOT-12? HSS J 2019;15:103-108.

34. Griffin DR, Parsons N, Mohtadi NG, Safran MR. A short version of the International Hip Outcome Tool (iHOT-12) for use in routine clinical practice. Arthroscopy 2012;28: 611-616. quiz 6-8.

35. Okoroha KR, Beck EC, Nwachukwu BU, Kunze KN, Nho SJ. Defining minimal clinically important difference and patient acceptable symptom state after isolated endoscopic gluteus medius repair. Am J Sports Med 2019;47:3141-3147.

36. Sutter R, Kalberer F, Binkert CA, Graf N, Pfirrmann CW, Gutzeit A. Abductor tendon tears are associated with hypertrophy of the tensor fasciae latae muscle. Skeletal Radiol 2013;42:627-633.

37. Agrawal V. Healing rates for challenging rotator cuff tears utilizing an acellular human dermal reinforcement graft. Int J Shoulder Surg 2012;6:36-44. 